\title{
émulations
}

\section{De la société en jeu au jeu comme société : un parcours dialectique}

\author{
Fanny Barnabé, Julien Bazile, Rémi Cayatte
}

Émulations - Revue de sciences sociales

2019, $\mathrm{n}^{\circ}$ 30, «Comment les jeux font-ils société. Contenus, pratiques et médiations ludiques ».

\section{Article disponible à l'adresse suivante}

https://ojs.uclouvain.be/index.php/emulations/article/view/barnabe

\section{Pour citer cet article}

Fanny Barnabé, Julien Bazile, Rémi Cayatte, « De la société en jeu au jeu comme société : un parcours dialectique », Émulations, n³0, Mise en ligne le 3 septembre 2019. DOI : 10.14428/emulations.030.01

Distribution électronique : Université catholique de Louvain (Belgique) : ojs.uclouvain.be

(C) Cet article est mis à disposition selon les termes de la Licence Creative Commons Attribution, Pas d'Utilisation Commerciale 4.0 International. http://creativecommons.org/licenses/by-nc/4.0/

Éditeur : Émulations - Revue de sciences sociales / Presses universitaires de Louvain https://ojs.uclouvain.be/index.php/emulations

ISSN électronique : $1784-5734$

$\frac{\text { PUL PRESSES }}{\text { UNIVERSITAIRES }}$ 


\title{
De la société en jeu au jeu comme société : un parcours dialectique
}

\author{
Fanny Barnabé1, Julien Bazile², Rémi Cayatte ${ }^{3}$
}

Le présent numéro interroge le vaste champ des relations entre jeux vidéo et société, en prenant pour porte d'entrée la question - volontairement polysémique - suivante : " comment les jeux font-ils société ?». La culture vidéoludique (ses œuvres et ses pratiques) s'inscrit toujours dans un contexte social donné qui en détermine largement le fonctionnement, les tensions internes, les évolutions, et qui, en retour, est lui-même représenté, travaillé, réfléchi par cette culture. Les relations et influences liant le jeu et le social peuvent donc prendre des visages multiples et des directions diverses, dont les textes de ce numéro rendent compte tant par la richesse de leurs objets que par la diversité de leurs approches. Nous tâcherons ici de retracer le parcours scientifique que ces regards croisés permettent d'effectuer : partant de la question de la place du jeu (et des joueurs·euses) dans la société contemporaine, nous passerons à celle de la représentation du social dans les jeux, pour enfin aborder la culture ludique comme étant, au-delà d'une figuration du social, un outil de médiation et de sociabilisation à part entière.

\section{Le jeu vu par la société}

Le jeu vidéo prend depuis quelques années une importance croissante dans notre paysage culturel et médiatique, et a largement transformé nos manières d'appréhender le monde qui nous entoure. Que l'on pense à l'amplitude de succès populaires tels que Fortnite (Epic Games, 2017)4, à la manière dont Pokémon GO (Niantic, 2016) a redéfini le rapport à la ville de ses millions de joueurs actifs, ou, plus généralement, à la généralisation des pratiques vidéoludiques chez un public de plus en plus large (depuis la « révolution casual » déjà décrite par Juul en 20105), il apparaît que les implications sociales du jeu ne peuvent plus se passer d'un examen scientifique et critique approfondi.

\footnotetext{
${ }^{1}$ Université de Liège, Liège Game Lab, Belgique.

2 Université de Lorraine, CREM, France, Université de Sherbrooke, Canada.

3 Université de Lorraine, CREM, France.

${ }^{4}$ Que d'aucuns vont jusqu'à assimiler à une nouvelle forme de réseau social. Voir l'article de Corentin Lamy, "De simple jeu à prototype de réseau social, comment "Fortnite" entend durer », Le Monde, 6 avril 2019. En ligne, consulté le 25 mai 2019. URL : https://www.lemonde.fr/pixels/ article/2019/04/06/de-simple-jeu-a-prototype-de-reseau-social-comment-fortnite-entend-durer_5446670_4408996.html.

${ }^{5}$ Ce dernier analyse en effet les mutations de la culture vidéoludique entraînées par l'apparition de consoles, supports et genres vidéoludiques plus accessibles et plus orientés « grand public » (la Wii de Nintendo, les jeux pour téléphones portables, les jeux de navigateur ou encore les « social games », par exemple).
} 
L'étude de cette inscription du jeu dans la société a longtemps été limitée à la question polarisée de son rejet ou de sa légitimation - le premier étant dicté par les reproches classiquement mobilisés pour décrier les cultures populaires ${ }^{6}$, tandis que la seconde est souvent motivée par l'attribution au jeu de vertus pédagogiques (Smaldone et al., 2016 ; Thorsteinsson et Niculescu, 2016) ou d'une propension exceptionnelle à susciter l'engagement de ses récepteurs ${ }^{7}$. Cependant, le développement et la diversification des sciences du jeu (ajoutés à l'omniprésence du jeu vidéo dans le quotidien du public) ont permis de dépasser cette étape de la "validation » sociale et morale du médium et de produire des analyses et critiques plus fines des nombreuses questions qu'il soulève.

Ces dernières années ont ainsi vu émerger, dans la recherche, dans la presse, mais aussi dans les discours des joueurs et joueuses, de nombreux débats touchant aux travers de l'industrie vidéoludique, à la place du politique dans les œuvres, ou encore au fonctionnement des communautés de "gamers». Récemment, plusieurs investigations menées par la presse spécialisée ${ }^{8}$ et projets de recherche ${ }^{9}$ ont mis en lumière le fonctionnement « toxique » de nombreux studios de développement, où se sont généralisées des conditions de travail précaires et sous pression, des méthodes de management confinant au harcèlement ${ }^{10}$ et des pratiques nocives telles que le « $\operatorname{crunch}^{11}{ }$ (Schreier, 2018).

D’autres thématiques sociales ont plutôt émergé de la prise de parole des joueurs et joueuses, à l'image de la fameuse controverse du Gamergate ${ }^{12}$. Tandis que certains utilisaient le hashtag \#gamergate pour dénoncer des cas de collusion entre journalistes de jeux vidéo et éditeurs, d'autres s'en servaient pour s'insurger contre l'introduction de thèmes politiques au sein de jeux (car ces messages contrevenaient, selon eux, à la nature ludique des œuvres), avec toutefois pour ligne commune de prendre pour cibles prioritaires les femmes du milieu (journalistes, développeuses, influenceuses, etc.). Le

\footnotetext{
${ }^{6}$ Reproches liés aux thèmes de la violence ou de l'addiction (Billieux et al., 2014).

7 Voir Alina Dizik, « Can Gaming at Work Make You More Productive? », BBC.com, 8 juillet 2016. En ligne, consulté le 25 mai 2019. URL : www.bbc.com/capital/story/20160707-can-gaming-at-workmake-you-more-productive.

${ }^{8}$ Voir l'imposant dossier réalisé en partenariat par le journal spécialisé Canard $P C$ et le site d'information généraliste Médiapart : Le Fou I. (2018), « Crunch Investigation, l'enquête de Médiapart et Canard PC », CanardPC.com. En ligne, consulté le 27 mai 2019. URL : https://www.canardpc.com/ online/crunch-investigation.

${ }^{9}$ Voir notamment les activités du groupe TETRIS («Territoires et trajectoires professionnelles dans l'industrie du jeu vidéo »), qui étudie le fonctionnement de l'industrie du jeu vidéo en France : TETRIS. En ligne, consulté le 24 juin 2019. URL : https://tetris.hypotheses.org/.

${ }^{10}$ Voir l'article de William Audureau : «Quantic Dream, un fleuron du jeu vidéo français aux méthodes de management contestées», Le Monde, 14janvier 2018. En ligne, consultéle27 mai2019. URL: https:// www.lemonde.fr/pixels/article/2018/01/14/quantic-dream-un-fleuron-du-jeu-video-francaisaux-methodes-de-management-contestees_5241506_4408996.html

${ }_{11}$ Période intense de travail précédant l'achèvement d'un projet. Voir l'article de Jason Schreir : «Inside Rockstar Games' Culture of Crunch », Kotaku. En ligne, consulté le 24 juin 2019. URL : https://kotaku. com/inside-rockstar-games-culture-of-crunch-1829936466.

12 Pour un aperçu de ce mouvement hétéroclite, voir l'émission « Gamergate : rébellion populaire, ou éruption réac ? », Arrêt sur images. En ligne, consulté le 27 mai 2019. URL : https://www.arretsurimages.net/emissions/gamergate-rebellion-populaire-ou-eruption-reac.
} 
mouvement du Gamergate, qui a donné lieu à de violentes campagnes de harcèlement contre ces personnalités féminines ${ }^{13}$, a ainsi été l'occasion de prendre la mesure du fond de sexisme qui traverse la culture vidéoludique ${ }^{14}$ et de mettre au jour les mécanismes de domination à l'œuvre dans la définition de ce qui peut ou non en faire partie.

En lien avec la controverse sur ce que sont ou devraient être les jeux vidéo mise en lumière par ce mouvement du Gamergate, la question de ce qui définit un « vrai » jeu et, par extension, un « vrai » joueur est encore réapparue, plus récemment, avec la sortie du jeu Sekiro: Shadows Die Twice (FromSoftware, 2019). Successeur des séries Dark Souls et Bloodborne, connues pour leur difficulté, ce jeu d'action a été critiqué par certains pour son manque d'accessibilité et défendu par d'autres comme le garant d'une culture vidéoludique exigeante, privilégiant l'art du gameplay sur les autres formes d'expression et se réservant aux véritables passionnés.

Ces différentes polémiques font apparaître une mutation essentielle du jeu vidéo : celui-ci ne peut plus se lire comme une pratique uniforme menée par un groupe d'amateurs homogènes (comme le loisir technologique d'une frange marginale de la population). Il est aujourd'hui devenu le terrain de luttes internes, de rapports de force entre une multitude de communautés et d'individus qui redéfinissent constamment, par leurs pratiques et leurs prises de position, les contours de ce qui peut être entendu par « vidéoludique».

C'est précisément à ce type de conflits identitaires que s'est intéressé David Peyron, en ouverture de ce numéro. Partant du constat (révélé notamment par l'enquête "Ludespace $»^{15}$ ) que la pratique du jeu vidéo s'est aujourd'hui massifiée et que le terme de « joueur » recouvre, dans les faits, une large variété d'identités et d'activités, le texte de Peyron s'interroge sur les stratégies mobilisées par les praticiens et praticiennes pour se construire et pour revendiquer une identité de "gamer ». À partir d'un terrain sociologique qualitatif, ce dernier met au jour les différentes manières par lesquelles joueurs et joueuses tâchent de démontrer leur « authenticité » et comment cette valeur d'authenticité est construite dans un contexte donné. Plusieurs critères sont ainsi dégagés des discours des enquêté·e·s, tels que la temporalité (il faut « avoir été là avant », aux « temps héroïques » où la pratique n'était pas encore partagée), l'intensité (il faut se distinguer des « antifigures » que sont le noob, le joueur débutant, et le casual, le joueur occasionnel), le « bon » goût (il faut avoir joué aux bonnes œuvres, privilégier certains genres de jeux et certains supports) et, plus largement, le rejet de tout ce qui est jugé « inauthentique ». À travers la définition du «vrai » et du « faux » se jouent, en somme,

${ }^{13}$ Voir l'article de William Audureau : « Une créatrice de jeux vidéo victime d'une vaste campagne de harcèlement en ligne », Le Monde, 22 août 2014. En ligne, consulté le 05 juin 2019. URL : https:// www.lemonde.fr/pixels/article/2014/08/22/une-creatrice-de-jeux-video-victime-d-une-vastecampagne-de-harcelement-en-ligne_4474760_4408996.html.

${ }^{14}$ Sur les questions de genres liées au jeu vidéo, voir, entre bien d'autres, les nombreuses publications du journal First Person Scholar. En ligne, consulté le 27 mai 2019. URL : http://www.firstpersonscholar.com/.

${ }^{15}$ Enquête dont l'objectif principal était de «saisir dans toute leur diversité sociologique et géographique les publics et les pratiques de jeux vidéo en France » (Boutet, Colón de Carvajal, Ter Minassian et Triclot, 2014). 
des processus de hiérarchisation culturelle et des rapports de pouvoir dont Peyron décrit les rouages et montre les implications sur la manière dont joueurs et joueuses se racontent.

En miroir de ce premier article qui replace le jeu vidéo dans un contexte culturel et social, quatre textes ont plutôt choisi de s'interroger sur la façon dont le social peut trouver sa place dans le jeu. Quelles sont les spécificités du dispositif ludique lorsqu'il évoque des enjeux sociaux et des événements présents ou passés ? Quels sont les moyens proprement ludiques de cette représentation de la société ? Et comment certains jeux parviennent-ils à concilier des impératifs de divertissement et d'interaction avec une volonté - parfois revendiquée - de remettre en question ou de conforter le rapport que nous avons à des problématiques sociétales (Bogost, 2007) ? C'est à ce type de questions que les textes suivants viennent apporter des réponses.

\section{La société vue par le jeu}

Plusieurs contributions présentes dans ce numéro participent à la déconstruction, opérée depuis plusieurs années, de l'idée selon laquelle le jeu est un espace hors du monde. Bien que singulier et hors de l'ordinaire, le « cercle magique » du jeu ${ }^{16}$ n'est pas un espace déconnecté strictement de la réalité (Consalvo, 2009). Il est, au contraire, tout à la fois un produit et un reflet du réel social, culturel, économique et politique ${ }^{17}$. Les espaces vidéoludiques sont des créations qui peuvent, par leur forme, traduire des valeurs ou exprimer des conceptions du monde, reproduire, perpétuer ou subvertir des systèmes de domination, des manières d'appréhender ou de s'approprier un territoire (Aarseth, 2014 ; Dyer-Witheford et De Peuter, 2009).

Les sciences du jeu s'inscrivent ici dans la continuité du constat établi par les études géographiques, architecturales ou urbanistiques, selon lequel concevoir un environnement revient à manier un code, à formuler un " message » à destination de celles et ceux qui l'habitent ou le traversent (Lynch, 1960). Il en va de même pour les environnements vidéoludiques. Loin d'être de simples habillages destinés à enjoliver des casse-têtes génériques, ces environnements sont porteurs de sens et invitent à repenser les manières par lesquelles joueurs et joueuses parcourent et s'approprient ces espaces.

La contribution de Guillaume Baychelier et José-Luis De Miras va dans ce sens en mobilisant la perspective de l'exploration touristique pour penser la présence du joueur ${ }^{18}$. Les auteurs y montrent comment les catégories conceptuelles servant à penser l'expérience touristique ("flux », « habitat », « transit ») rendent intelligibles les environnements vidéoludiques comme étant davantage que de simples « terrains de jeu » : ils sont

\footnotetext{
${ }^{16}$ Notion conçue par Johan Huizinga (1951 [1938]), mais principalement développée par Katie Salen et Eric Zimmerman (2004), pour définir le jeu comme un espace clôturé, séparé du quotidien et régi par des règles particulières, n'ayant pas cours au-delà du « cercle ».

17 Voir Éric Zimmerman, "Jerked Around by the Magic Circle - Clearing the Air Ten Years Later », Gamasutra, 7 février 2012. En ligne, consulté le 30 mai 2019. URL : https://www.gamasutra.com/ view/feature/135063/jerked_around_by_the_magic_circle_.php.

18 Sur le sujet, voir également Andrzej Zarzycki (2016).
} 
des lieux de vie sociale, d'expression identitaire, d'investissement citoyen ou d'exploration dépaysante. L'étude de cas menée sur le jeu vidéo Silent Hill (Konami, 1999) illustre ce dernier point, et fournit un exemple de la façon dont l'exploration vidéoludique peut être une expérience dépaysante qui nécessite (et c'est là tout son intérêt) de l'attention, de la scrutation et de la témérité (Bazile, 2018) ou de la prudence que le joueur ou la joueuse doit déployer.

Plus encore, l'étude des sociétés historiques apparaît fournir des clefs qui permettent de mieux comprendre les représentations dont elles peuvent faire l'objet dans les jeux. En cela, le village d'Euzéro, cas d'étude dans l'article de Guillaume Grandjean au sein de ce numéro, constitue un contre-exemple au concept de « négligence étudiée » importé par l'auteur. Issu de la rhétorique classique, ce dernier désigne le « désordre phénoménologique apparent censé reproduire l'organisation "naturelle" (non rationnelle) des espaces réels, tout en masquant la répartition logique et rigoureuse des éléments de design nécessaires au système de jeu ». L'organisation des espaces sociaux réels peut traduire le dessein d'efficience et la recherche du fonctionnel de ses concepteurs (comme c'est le cas dans l'organisation urbaine coloniale ; Burnard et Garrigus, 2016 ; Sainton, 2012), mais aussi sa nature organique, spontanée et humaine (Szymanezyk et al., 2011).

Objets issus d'une industrie mondialisée, les jeux vidéo prennent parfois explicitement pour sujet le réel historique. Particulièrement, la question de la place de la réalité historique et du lien à l'actualité dans la mise en jeu du conflit ethnique est abordée par Sébastien Wit à travers une étude du jeu Tactics Ogre (Quest, 1995). Cette publication s'ajoute à celles qui, depuis quelques années, font rencontrer études postcoloniales et études vidéoludiques, relevant la persistance du paradigme ethnico-colonial dans les productions contemporaines (Mukherjee, 2017; 2018 [2016]). Elle met en lumière le fait que le jeu vidéo peut être un médium qui ne décrit pas seulement la complexité du réel historique, mais l'incarne également, en tant que production dont le contenu est apprécié entre fiction artistique, pressions contextuelles dues à l'environnement socio-économique contemporain et recherche de référence historique (Ayers, 2001). Comme le mentionne Sébastien Wit à l'issue de son article : «L'univers est un système dynamique au sein duquel rien n'est réductible à des oppositions binaires ».

Une deuxième contribution du présent numéro s'intéresse à la question de la représentation identitaire dans les jeux, en particulier lorsqu'il s'agit d'identités minoritaires ou « subalternes » (Gajjala, 2012 ; Hammar, 2015 ; 2016 ; Lauro, 2017). Julien Hocine et Patrick Deslauriers présentent les résultats d'une étude menée sur les représentations de l'identité autochtone dans le jeu Kisima Innitchuna (Never Alone) (Upper One Games, 2014), résultats qui invitent à considérer la façon dont le jeu vidéo influence le regard des autochtones de l'Arctique sur eux-mêmes. On retrouve dans la dialectique « regard de l’intérieur/regard de l'extérieur » mentionnée par les auteurs en conclusion, un écho au concept de « double-conscience » (« double consciousness ») formulé par W.E.B. Du Bois et repris par Paul Gilroy (2017) pour exprimer le conflit interne présent à l'esprit d'individus qui intériorisent les représentations issues de la société dans laquelle ils 
se trouvent en tant que subordonnés. L'étude interroge aussi le potentiel du médium vidéoludique en tant que vecteur d'expression et en tant qu'outil de préservation du patrimoine culturel - ce qui, comme nous le verrons, n'est pas sans lien avec les textes évoqués au point suivant.

Ces quatre contributions donnent en somme à voir comment le jeu porte un regard sur la société et comment l'environnement social, historique, culturel et politique influence la conception des objets (vidéo)ludiques. En retour, ces articles ne manquent également pas de s'interroger sur les effets que leurs représentations du social ont sur les sociétés et communautés dans lesquelles elles s’inscrivent.

\section{Médiation et sociabilisation par le jeu}

Loin de n'être qu'une représentation « hors sol », le jeu constitue également un espace de sociabilité à part entière autant qu'un moyen d'agir sur la société. Le texte d'Esteban Giner adopte une perspective similaire en partant de l'analyse de Night In The Woods (Infinity Fall, 2017) tout en envisageant comment celui-ci peut affecter son récepteur. À travers la représentation d'une ville fictive des États-Unis frappée par la récession économique et la narration des difficultés rencontrées par ses habitants, Night In The Woods porte en effet un discours critique sur la société contemporaine et, plus spécifiquement, sur des problématiques faisant partie du quotidien de ses auteurs et d'une partie de son public (perte d'emploi et difficultés financières suite à la crise des subprimes notamment). À l'aide de modèles théoriques empruntés à Sébastien Genvo (2013) et Rémi Cayatte (2018), complétés par des concepts issus de la cybernétique (Galloway, 2006) et des sciences de l'éducation (Houssaye, 1993), Giner révèle les mécanismes permettant au jeu de mettre en place une pluralité de discours : si certains contenus sont véhiculés sur un mode plutôt " persuasif » (Bogost, 2007), ceux-ci alternent avec des séquences plus « expressives »(Genvo, 2016). Au-delà de cette étude de cas, le modèle d'analyse proposé dans cet article permet d'étudier le jeu vidéo non seulement en tant que contenu ou support d'une pratique, mais aussi en tant qu'acte de communication qui laisse place à la controverse, autre notion développée par l'auteur.

Cette considération du jeu comme acte communicationnel est au cœur des initiatives qui, de plus en plus, utilisent le médium vidéoludique comme outil de médiation ou d'expression citoyenne. On ne compte plus les musées (Doduik, 2018), associations (Bonvoisin et Culot, 2015), écoles (Gilson et Préat, 2018) ou institutions culturelles (Delbouille, 2013) qui voient dans les jeux des intermédiaires précieux, capables de se faire tant les vecteurs de messages (artistiques, culturels, politiques, etc.) que les supports d'une prise de parole personnelle, permettant aux utilisateurs de s'approprier par le jeu le monde qui les entoure. L'entretien avec Sébastien Genvo présent dans ce numéro revient précisément sur ce pouvoir expressif du jeu vidéo. Le chercheur s’y interroge sur les manières par lesquelles «le jeu véhicule des normes culturelles, des représentations du monde et des valeurs », mais aussi sur la façon spécifique dont il "permet aux individus d'exprimer leurs propres représentations du monde et valeurs ». En 
présentant son dernier projet de recherche-création, Genvo illustre davantage cette notion de « jeu expressif » et l'importance de concevoir des jeux mettant en scène des thématiques intimes et des problèmes quotidiens (tout en révélant les difficultés que ce type de propos pose en termes de game design).

Enfin, dans le prolongement de cette démarche réflexive, Dominic Arsenault clôture le présent numéro dans une conclusion qui revient sur l'intérêt qu'a revêtu, pour lui, la lecture des six articles. Cette conclusion permet notamment de souligner, en marge de la problématique des rapports entre jeu et société, la nature profondément pluridisciplinaire des différentes contributions et plus largement des sciences du jeu dans leur ensemble. Au-delà des thématiques développées dans le présent numéro, c'est donc aussi la place des travaux portant sur le jeu dans la société et dans le monde académique que cet ensemble de textes permet, en filigrane, de (re)penser.

\section{Bibliographie}

Aarseth E. (2014), " I Fought the Law: Transgressive Play and the Implied Player », in N. Segal, D. Koleva (dir.), From Literature to Cultural Literacy, London, Palgrave Macmillan UK, p. 180-188.

Ayers E. L. (2001), «The Pasts and Futures of Digital History », History News, vol. 56, n 4 , p. 5-9.

BAZILE J. (2018), « Ludoformer Lovecraft : Sunless Sea comme mise en monde du mythe de Cthulhu », Sciences du jeu, nº 9. En ligne, consulté le 30 mai 2019. URL : http:// journals.openedition.org/sdj/996.

Billieux J., Deleuze J., Griffiths M., Kuss D. (2014), « Internet Gaming Addiction: The Case of Massively Multiplayer Online Role Playing Games », in N. El-Guebaly, M. Galanter, G. Carrá (dir.), The Textbook of Addiction Treatment: International Perspectives, New York, Springer, p. 1515-1525.

Bogost I. (2007), Persuasive Games: The Expressive Power of Videogames, Cambridge, The MIT Press.

Bonvoisin D., Culot M. (2015), Éducation aux médias et jeux vidéo : des ressorts ludiques à l'approche critique, Bruxelles, Média Animation. En ligne, consulté le 24 juin 2019. URL : https://media-animation.be/IMG/pdf/media_animation_-_education_aux_ medias_et_jeux_video.pdf.

Boutet M., Colón de Carvajal I., Ter Minassian H., Triclot M. (2014), « Au-delà du virtuel : interactions sociales et spatiales dans et autour d'un univers vidéoludique », MEI Médiation et information, $\mathrm{n}^{\circ}$ 37, p. 103-116. En ligne, consulté le 27 mai 2019. URL : https://halshs.archives-ouvertes.fr/halshs-00851446/file/Ter_minassian_et_alii_A_ paraA_tre_dans_MEI_37_.pdf.

Burnard T., Garrigus J. (2016), The Plantation Machine: Atlantic Capitalism in French SaintDomingue and British Jamaica, Philadelphia, University of Pennsylvania Press. 
Cayatte R. (2018), «Temps de la chose-racontée et temps du récit vidéoludique : comment le jeu vidéo raconte? », Sciences $d u$ jeu, n 9. En ligne, consulté le 31 mai 2019. URL : https://journals.openedition.org/sdj/936.

Consalvo M. (2009), « There is No Magic Circle », Games and Culture, vol. 4, n 4, p. 408417. En ligne, consulté le 30 mai 2019. URL : https://www.academia.edu/654444/ There_is_no_magic_circle.

Delboullle J. (2013), Le jeu vidéo, un médium en voie de légitimation ? Étude de réception auprès du grand public en Belgique francophone. Mémoire de master en Information et Communication, Liège, Université de Liège. En ligne, consulté le 24 juin 2019. URL : https://orbi.uliege.be/handle/2268/206382.

Doduik N. (2018), « Entre médiation culturelle et médiation ludique : étude d’un jeu comme dispositif de médiation dans un musée ", Communication présentée au colloque Entre le jeu et le joueur : écarts et médiations, Liège, Belgique. En ligne, consulté le 30 mai 2019. URL : https://www.youtube.com/watch?v=Knqvzx7v0dQ.

Dyer-Witheford N., De Peuter G. (2009), Games of Empire: Global Capitalism and Video Games, Minneapolis, University of Minnesota Press.

GajJALA R. (2012), Cyberculture and the Subaltern: Weavings of the Virtual and Real, Lanham, Lexington Books.

Galloway A. R. (2006), Gaming: Essays on Algorithmic Culture, Minneapolis, University of Minnesota Press.

Genvo S. (2013), « Penser les phénomènes de ludicisation à partir de Jacques Henriot », Sciences $d u$ jeu, $\mathrm{n}^{\circ}$ 1. En ligne, consulté le 14 juillet 2019. URL : https://journals. openedition.org/sdj/251

Genvo S. (2016), « Defining and Designing Expressive Games: The case of Keys of a Gamespace », Kinephanos, ${ }^{\circ}$ special : Exploring the Frontiers of Digital Gaming. En ligne, consulté le 31 mai 2019. URL : https://www.kinephanos.ca/2016/defining-anddesigning-expressive-games/.

Gilroy P. (2017), L’Atlantique noir : modernité et double conscience, Paris, Amsterdam.

Gilson G., PréAt C. (2018), « Les jeux (vidéo) en contexte scolaire : quand les cultures ludiques s'invitent en classe », in Actes du colloque Ludovia 2018 : Innovation (s)/ Institution(s), Salvagnac, Culture Numérique. En ligne, consulté le 24 juin 2019. URL: http://culture.numerique.free.fr/publications/ludo18/Gilson_Preat_Ludovia_2018. pdf.

Hammar E. L. (2015), « Ethical Recognition of Marginalized Groups in Digital Games Culture ", Communication présentée au colloque DiGRA 2015: Diversity of Play: Games - Cultures - Identities, Lüneburg, Allemagne. En ligne, consulté le 30 mai 2019. URL : http://www.digra.org/wp-content/uploads/digital-library/215_ Hammar_Ethical-Recognition-of-Marginalized-Groups-in-Digital-Games-Culture. pdf. 
Hammar E. L. (2016), « Counter-hegemonic Commemorative Play: Marginalized Pasts and the Politics of Memory in the Digital Game Assassin's Creed: Freedom Cry », Rethinking History, vol. 21, n 3, p. 372-395. En ligne, consulté le 12 juillet 2019. URL : https://www.tandfonline.com/doi/full/10.1080/13642529.2016.1256622.

Houssaye J. (dir.) (1993), Pédagogie : une encyclopédie pour aujourd'hui, Montrouge, ESF Éditeur.

Huizinga J. (1951 [1938]), Homo Ludens. Essai sur la fonction sociale du jeu, Paris, Gallimard.

Juvl J. (2010), A Casual Revolution: Reinventing Video Games and Their Players, Cambridge, MIT Press.

LAURo S. J. (2017), «Digital Saint-Domingue: Playing Haiti in Videogames », sx archipelagos, $\mathrm{n}^{\circ}$ 2. En ligne, consulté le 30 mai 2019. URL : http://smallaxe.net/sxarchipelagos/ issue02/playing-haiti.htm.

Lynch K. (1960), The Image of the City, Cambridge, The MIT Press.

MukHerJEe S. (2017), «Videogames and Post colonialism: An Introduction», in S. MukHERJeE, Videogames and Postcolonialism, Cham, Springer International Publishing, p. 1-28. En ligne, consulté le 30 mai 2019. URL : http://link.springer.com/10.1007/978-3-31954822-7_1.

MukherJeE S. (2018 [2016]), « Playing Subaltern: Video Games and Postcolonialism », Games and Culture, vol. 13, n 5. En ligne, consulté le 30 mai 2019. URL : https://doi. org/10.1177/1555412015627258.

SAINTON J.-P. (2012), Histoire et civilisation de la Caraïbe. Tome 2 : Le temps des matrices, économie et cadres sociaux du long XVIII siècle, vol. 2, Paris, Karthala.

Salen K., Zimmerman E. (2004), Rules of Play. Game Design Fundamentals, Cambridge, MIT Press.

Smaldone R. A., Thompson C. M., Evans M., Voit W. (2016), « Teaching Science Through Video Games », Nature Chemistry, vol. 9, p. 97-102.

Szymanezyk O., Dickinson P., Duckett T. (2011), « From Individual Characters to Large Crowds: Augmenting the Believability of Open-world Games through Exploring Social Emotion in Pedestrian Groups », DiGRA 2011: Think Design Play, Utrecht, Netherlands. En ligne, consulté le 30 mai 2019. URL : http://eprints.lincoln. ac.uk/4662.

Thorsteinsson G., Niculescu A. (2016), « Pedagogical Insights into the Use of Minecraft within Educational Settings », Studies in Informatics and Control, vol. 25, $\mathrm{n}^{\circ}$ 4, p. 507516.

ZARZYcki A. (2016), "Epic Video Games: Narrative Spaces and Engaged Lives », International Journal of Architectural Computing, vol. 14, $\mathrm{n}^{\circ}$ 3, p. 1-11. 


\title{
émulations
}

\section{De la société en jeu au jeu comme société : un parcours dialectique}

\author{
Fanny Barnabé, Julien Bazile, Rémi Cayatte
}

Émulations - Revue de sciences sociales

2019, $\mathrm{n}^{\circ}$ 30, «Comment les jeux font-ils société. Contenus, pratiques et médiations ludiques ».

\section{Article disponible à l'adresse suivante}

https://ojs.uclouvain.be/index.php/emulations/article/view/barnabe

\section{Pour citer cet article}

Fanny Barnabé, Julien Bazile, Rémi Cayatte, « De la société en jeu au jeu comme société : un parcours dialectique », Émulations, n³0, Mise en ligne le 3 septembre 2019. DOI : 10.14428/emulations.030.01

Distribution électronique : Université catholique de Louvain (Belgique) : ojs.uclouvain.be

(C) Cet article est mis à disposition selon les termes de la Licence Creative Commons Attribution, Pas d'Utilisation Commerciale 4.0 International. http://creativecommons.org/licenses/by-nc/4.0/

Éditeur : Émulations - Revue de sciences sociales / Presses universitaires de Louvain https://ojs.uclouvain.be/index.php/emulations

ISSN électronique : $1784-5734$

$\frac{\text { PUL PRESSES }}{\text { UNIVERSITAIRES }}$ 